\title{
Case-Control Study of Diet and Prostate Cancer in a Rural Population of Faisalabad, Pakistan
}

\author{
Muhammad Naeem Bashir ${ }^{1 *}$, Muhammad Akram Malik²
}

\begin{abstract}
Background: The effects of diet on epidemiology of prostate cancer are inconclusive. Therefore a hospitalbased, case-control study was conducted in a rural population of Faisalabad, Pakistan, to examine the impact of dietary factors on risk of cancer development. Materials and Methods: This study was based on 102 confirmed cases of prostate cancer and 204 normal controls. Logistic regression was used to estimate odds ratios and $95 \%$ confidence intervals for odds ratios to evaluate the relationship between prostate cancer and diet. Results: Consumption of red meat and fat items significantly increased the prostate cancer risk having odds ratios and 95\% confidence intervals of $3.41 ; 1.46-7.96$ and $2.45 ; 1.17-5.15$, respectively. On the other hand, more consumption of vegetables, fluid intake and fruit significantly decreased the prostate cancer risk (odd ratios and corresponding $95 \%$ confidence intervals of $0.21 ; 0.10-0.44,0.10 ; 0.05-0.19$ and $0.09 ; 0.03-0.23$, respectively. Conclusions: The present study supports the hypothesis that frequent consumption of red meat and fat items may increase prostate cancer risk while more intake of fruit, vegetables and fluid intake may protect against prostate cancer in the relatively low risk group in rural Pakistan.
\end{abstract}

Keywords: Prostate cancer - diet - fat items - fruit - odds ratio - rural population - Pakistan.

Asian Pac J Cancer Prev, 16 (6), 2375-2378

\section{Introduction}

Prostate cancer is the commonly diagnosed malignancy among males in developed countries. Worldwide, incidence rates have been increased significantly through early 1990's when screening with prostate specific antigen was introduced (Hsing et al., 2006). Prostate cancer is the second leading cancer among Americans. It is estimated that almost 220,800 new cases of prostate cancer will be diagnosed in the United States in 2015 which is $26 \%$ of all the malignancies among men and the estimated number of deaths will be about 27,540. A man's life time risk of prostate cancer is one out of seven (Siegel et al., 2015). The morbidity and mortality rates of prostate cancer are remarkably different in various geographic regions with highest rate in northern America and the lowest in Asia. During 1998-2002, the age standardized incidence rate in Karachi (Pakistan) was 10.1 per 100,000 men (Bhurgri et al., 2009). This is comparable to Asia- Pacific region 9.9 per 100,000 but much lower than the whole world 32.8 per 100,000 men (Baade et al., 2013).

The large global variation in both morbidity and mortality rates has suggested that environmental factors such as diet may affect prostate cancer risk, but no dietary risk factors have been definitely established. The findings on the association of fruit and vegetable intake and prostate cancer have not been consistent (Meng et al., 2013).
Askari et al (2014) recently observed in their study that increased consumption of fruit and vegetables has been associated with reduced risk of prostate cancer. Low fat diet, high intake of vegetables and fruit were found to be protective against prostate cancer risk (Shahar et al., 2011). More consumption of fat items, particularly red meat and dairy products was positively associated with prostate cancer risk in many studies (Vlajinac et al., 2010; Masko et al., 2012).

The purpose of the present study was therefore to evaluate the relationship between dietary factors and prostate cancer risk among Pakistani population.

\section{Materials and Methods}

This is a hospital-based case control study, conducted in three main hospitals of Faisalabad (Pakistan) during September, 2012 to August, 2013. The selected hospitals were PINUM Cancer hospital, Punjab Medical College Allied hospital and Madina Teaching Hospital, Faisalabad. The study was based on 102 confirmed cases of prostate cancer and 204 normal controls. All the participants were interviewed face to face in the hospitals using the structured questionnaire. The questionnaire included information about dietary factors such as fluid intake, consumption of red meat, fat items and use of fruits and vegetables. 
Table 1. Characteristics of Cases and Controls for Different Factors

\begin{tabular}{|c|c|c|c|c|c|}
\hline Factor & Characteristics & Cases & $\%$ & Controls & $\%$ \\
\hline Total & & 102 & & 204 & \\
\hline Mean age & & 68 year & & 53 years & \\
\hline \multirow[t]{2}{*}{ Fluid consumption } & Less than 7 glasses daily & 81 & 79.4 & 46 & 22.5 \\
\hline & 7 or more glasses daily & 21 & 20.6 & 158 & 77.5 \\
\hline \multirow[t]{3}{*}{ Vegetables used (no of days in a week) } & Low(0 to 2 days $)$ & 82 & 80.4 & 99 & 48.5 \\
\hline & Normal(3 to 5 days) & 20 & 19.6 & 105 & 51.5 \\
\hline & Excessive(6 to 7 days) & 0 & 0 & 0 & 0 \\
\hline \multirow[t]{3}{*}{ Red meat(no of days in a week) } & Low (0 to 2 day) & 69 & 67.6 & 177 & 86.7 \\
\hline & Normal(3 to 5 days) & 33 & 32.4 & 27 & 13.3 \\
\hline & Excessive(6 to 7 days) & 0 & 0 & 0 & 0 \\
\hline \multirow[t]{6}{*}{ Fat items (no of days in a week) } & Low (0 to 2 days) & 62 & 60.8 & 154 & 75.5 \\
\hline & Normal(3 to 5 days) & 40 & 39.2 & 50 & 24.5 \\
\hline & Excessive( 6 to 7 days) & 0 & 0 & 0 & 0 \\
\hline & Low (0 to 2 days) & 95 & 93.1 & 110 & 53.9 \\
\hline & Normal(3 to 5 days) & 7 & 6.9 & 94 & 46.1 \\
\hline & Excessive(6 to 7 days) & 0 & 0 & 0 & 0 \\
\hline \multirow[t]{3}{*}{ Use of fruit (no of days in a week) } & Low (0 to 2 days) & 95 & 93.1 & 110 & 53.9 \\
\hline & Normal(3 to 5 days) & 7 & 6.9 & 94 & 46.1 \\
\hline & Excessive(6 to 7 days) & 0 & 0 & 0 & 0 \\
\hline
\end{tabular}

Table 2. Correct Classification and Misclassification of Subjects

\begin{tabular}{lcccc}
\hline Observed & \multicolumn{3}{c}{ Predicted Prostate Cancer } & Percentage \\
& & No & Yes & Correct \\
\hline Prostate Cancer & No & 182 & 22 & 89.2 \\
& Yes & 30 & 72 & 70.6 \\
Overall Percentage & & & & 83 \\
\hline
\end{tabular}

Consumption of red meat, fat items and use of fruits and vegetables ws taken as ordinal variables having three categories of low, normal and excessive i. e. Low=less than 3 days in a week, Normal=3 to 5 days in a week and Excessive $=6$ to 7 days in a week .

In descriptive analysis bivariate tables were used for explaining the count of each possible risk factor for both cases and controls. In analytical approach, the binary logistic regression model was used to estimate the odds ratios and $95 \%$ confidence interval for odd ratios to assess the relationship between diet and prostate cancer by using SPSS version 16.0 software. A variable is considered to be statistically significant if the P-value was less than 0.05 . The goodness of fit for the model was evaluated using the Omnibus and Hosmer- Lemeshow test.

\section{Results}

This study was based on 306 individuals, including 102 confirmed cases of prostate cancer and 204 normal controls. Table 1 shows the characteristics of cases and controls with respect to different risk factors. The mean age of cases and controls was 68 years and 53 years respectively. Age could not be matched because there were fewer people who visited the hospitals in older age as controls than cases. The proportion of men using frequently red meat and fat items was higher in cases than controls. On the other hand consumption of fruit and vegetables were lower in cases than controls. Fluid consumption was almost four times lower in cases than controls.

From Table 2, it was observed that out of 204 controls $182(89.2 \%)$ were correctly predicted as controls while out of 102 prostate cancer cases $72(70.6 \%$ ) were correctly predicted as cases. The overall numbers (percentages) of correctly classified and misclassified of subjects were 254 $(83.0 \%)$ and $52(17.0 \%)$, respectively. The percentage of correct classification was high, which means that the fitted model was acceptable.

Table 3 summarizes data for the relationship between different dietary factors and prostate cancer in terms of odd ratios and $95 \%$ confidence intervals. In this multivariate analysis, we observed that consumption of red meat was strongly associated with increased risk of prostate cancer (OR: 3.413, 95\%CI: 1.464-7.959, $\mathrm{p}=0.004$ ). The consumption of fat items was also linked to a more than two-fold enhanced risk of prostate cancer (OR: 2.454, 95\%CI: $1.171-5.145, \mathrm{p}=0.017)$.

Fluid intake was associated with a clearly decreased risk of prostate cancer (OR: 0.101,95\%CI: $0.0528-0.198$, $\mathrm{p}=0.017)$. The risk of prostate cancer tends to be reduced with frequent use of fruit and vegetables (OR: 0.087, 95\%CI: $0.034-0.227, \mathrm{p}=0.000)$ and (OR: $0.214,95 \% \mathrm{CI}$ : $0.104-0.442, \mathrm{p}=0.000)$ respectively.

Table 3. Association between different Factors and Prostate Cancer Risk in Logistic Regression Model

\begin{tabular}{|c|c|c|c|c|c|c|c|}
\hline \multirow[t]{2}{*}{ Factors } & \multirow[t]{2}{*}{ B } & \multirow[t]{2}{*}{ S.E. } & \multirow[t]{2}{*}{ Wald } & \multirow[t]{2}{*}{ Sig. } & \multirow[t]{2}{*}{ (OR) } & \multicolumn{2}{|c|}{$95 \% \mathrm{CI}$} \\
\hline & & & & & & Lower & Upper \\
\hline Fluid intake & -2.29 & 0.341 & 45.171 & 0.000 & 0.101 & 0.052 & 0.198 \\
\hline Consumption of red meat & 1.228 & 0.432 & 8.059 & 0.004 & 3.413 & 1.464 & 7.959 \\
\hline Fat item consumption & 0.898 & 0.378 & 5.651 & 0.017 & 2.454 & 1.171 & 5.145 \\
\hline Use of fruit & -2.438 & 0.489 & 24.906 & 0.000 & 0.087 & 0.034 & 0.227 \\
\hline Vegetables used & -1.542 & 0.37 & 17.341 & 0.000 & 0.214 & 0.104 & 0.442 \\
\hline
\end{tabular}




\section{Discussion}

Prostate cancer is the most frequently occurring cancer among men in Pakistan. It is one of the third leading sites of cancers in men with a ratio of about $7 \%$ of all malignancies (Ahmad et al., 2009). Dietary choices are influenced by cultural norms, economics and religious attachment. The dietary patterns can vary based on social class, residence, income level, education and disease and health status. Pattern of diet may differ between urban and rural area. For example, red meat consumption and use of dairy products are more dominant in rural areas. There is also wide variation in fruit and vegetable consumption.

In this study, dietary factors such as consumption of red meat, fat items, fluid intake, use of fruit and vegetables were evaluated and compared with previous studies from all over the world.

In the present study, the consumption of red meat and fat items has shown a statistically significant increase in the risk of prostate cancer. A cohort study- Physician's Health Study (Song et al., 2013) found an association between whole milk and the risk of fatal prostate cancer and worse survival in old men. Mahmood et al .(2012) found in their study that risk of developing prostate cancer was about 12 times higher with the consumption of red meat and dairy products. Intake of whole milk was strongly associated with increased risk of prostate cancer (HR: 2.15, 95\%CI: 1.28-3.60) (Petterson et al., 2012).

Joshi et al (2012) observed that high intake of red meat cooked at higher temperature was positively associated with advanced prostate cancer risk. Saleem et al (2011) observed in their study in Iraq that prostate cancer is significantly higher with increasing consumption of fat items. Lee et al (1998) conducted a case-control study in China to evaluate the association between dietary factors and prostate cancer risk. The data showed that both saturated and unsaturated fats are positively associated with higher risk of prostate cancer having odds ratios and 95\% confidence interval (OR: 3.6, CI: 1.8-7.7) and (OR: 2.9, CI: 1.5-5.7) respectively. More consumption of meat and fat items has been associated with increased risk of prostate cancer in several studies (Sonoda et al., 2004; Tyagi et al., 2010; Hardin et al., 2011; Masko et al., 2012).

Our study has shown that fluid intake significantly decreased the risk of prostate cancer. It means that a person, who consumes 7 or more glasses of any type of fluid per day, has almost ten times protection against prostate cancer. Bashir et al (2014) found in their study that prostate cancer tends to be reduced with frequent consumption of fluid intake (OR: 0. 052, 95\%CI: 0.024$0.115)$.

In this study consumption of fruit and vegetables has been associated with reduced risk of prostate cancer. Askari et al (2014) conducted a retrospective study in Iran to evaluate the association between fruit and vegetables and prostate cancer risk. They found a significantly decreased risk of prostate cancer for intake of fruit and vegetables (OR: 0.30, CI: 0.06-0.40) and (OR: 0.31, CI: $0.02-0.21)$ respectively. Consumption of leafy vegetables are inversely associated with prostate cancer risk (OR: 0.66, CI: 0.46-0.96) (Hardin et al., 2011).
A Meta-analysis was conducted by Zhou et al (2013), investigated the relationship between consumption of vegetables and prostate cancer risk. A total of 9 epidemiological studies comprising of 3 prospective cohort and 6 case- control studies were incorporated in this analysis. They found statistically significant reduced risk of prostate cancer for intake of allium vegetables (OR: 0.82, 95\%CI: 0.70-0.97). Sunny et al (2005) also observed in their study that low fat diet rich in vegetables and fruit may decreased the prostate cancer risk. Several studies have also shown that the consumption of fruit and vegetables decreased the risk of prostate cancer (Saleem et al., 2011; Liu et al., 2012; Mahmood et al., 2012).

Unlike the results of this study, some of the studies did not show a protective effect for vegetables and fruit consumption against prostate cancer risk. For instance, in a meta-analysis by Meng et al.(2013), they did not show a significant protective effect for vegetables and fruit against prostate cancer risk. Umesawa et al (2014) evaluated the association of vegetables intake and carotenes with risk of prostate cancer in Japan. They found that vegetable intake was not statistically associated with prostate cancer risk, however alpha-carotene intake is associated with lower risk of prostate cancer among Japanese men.

In conclusion, the case control study suggested that more consumption of fat items and use of red meat could be considered as probable risk factors for prostate cancer in Pakistani men. In addition, fluid intake and frequent use of fruit and vegetables may protect against the disease.

\section{Acknowledgements}

We are thankful to all subjects and their relatives for their cooperation. We are also grateful for the help and facilitation of all medical and paramedical staff of Punjab Medical College Allied Hospital, PINUM Cancer Hospital and Madina Teaching Hospital Faisalabad (Pakistan).

\section{References}

Ahmad Z, Qureshi A, Idrees R, Aftab K (2009). Prostatic carcinoma: a Pakistani perspective. Asian Pac J Cancer Prev, 10, 323-4.

Askari F, Parizi M K, Jessri M, Rashidkhani B (2014). Fruit and vegetables intake in relation to prostate cancer in Iranian men: A case control study. Asian Pac J Cancer Prev, 15, 5223-27.

Baade P D, Youlden D R, Cramb S M, Dunn J, Gardinar R A (2013). Epidemiology of prostate cancer in the Asia-Pacific region. Prostate Int, 1, 47-58.

Bashir M N, Ahmad M R, Malik A (2014). Risk factors of prostate cancer: a case- control study in Faisalabad, Pakistan. Asian Pac J cancer Prev, 15, 10327-30.

Bhurgri Y, Kayani N, Pervez S, et al (2009). Incidence and trends of prostate cancer in Karachi South, 1995-2002. Asian Pac $J$ Cancer Prev, 10, 45-8.

Hardin J, Cheng I, Witte J S (2011). Impact of consumption of vegetables, fruit, grain and high glycemic index food on aggressive prostate cancer risk. Nutr Cancer, 63, 860-72.

Hsing A W, Chokkalingam A P (2006). Prostate cancer epidemiology. Front Biosci, 11, 1388-413.

Joshi A D, Corral R, Catsburge, et al (2012). Red meat and poultry cooking practices, genetic susceptibility and risk 
Muhammad Naeem Bashir and Muhammad Akram Malik

of prostate cancer: result from a multiethnic case- control study. Carcinogenesis, 33, 2108-18.

Lee M M, Wang R T, Hsing AW, et al (1998). Case-control study of diet and prostate cancer in China. Cancer Cause Control, 9, 545-52.

Liu B, Mao Q, Cao M, Xie L (2012). Cruciferous vegetables intake and risk of prostate cancer: a meta-analysis. Int $J$ Urol, 19, 134-41.

Mahmood S, Qasmi G, Ahmad A, et al (2012). Lifestyle factors associated with the risk of prostate cancer among Pakistani men. J Ayub Med Coll Abbotabad, 24, 122-26.

Mask E M, Allott E H, Freedland S J (2012). The relationship between nutrition and prostate cancer: Is more always better? Eur Urol, 63, 810-20.

Meng H, Hu W, Chen Z, Shen Y (2013). Fruit and vegetables intake and prostate cancer risk: a meta-analysis. Asia Pac J Clin Oncol, 10, 133-40.

Salem S, Salahi M, Mohseni M (2011). Major dietary factors and prostate cancer risk: a prospective multicenter case control study. Nutr Cancer, 63, 21-27.

Sunny L (2005). A low fat diet rich in fruits and vegetables may reduce the risk of developing prostate cancer. Asian Pac $J$ Cancer Prev, 6, 490-6

Siegel R, Miller K D, Jemal A (2015). Cancer statistics. CA: Cancer J Clin, 65, 5-29.

Shahar S, Shafurah S, Hassan Sherazi NS, et al (2011). Role of diet, lifetime physical activity and oxidative DNA damage in the occurrence of prostate cancer among men in Klang Valley, Malaysia. Asian Pac J Cancer Prev, 12, 605-11.

Petterson A, Kasperzyk J L, Kenfield S A, et al (2012). Milk and dairy consumption among men with prostate cancer and risk of metastases and prostate cancer death. Cancer Epidemiol Biomarker Prev, 21, 428-36.

Song Y, Chavarro J E, Cao Y, Qiu W, et al (2013). Whole milk intake is associated with prostate cancer- specific mortality among US. male Physicians. J Nutt, 143, 189-96.

Sonoda T, Nagata Y, Mori, M. (2004). A case-control study of diet and prostate cancer in Japan: possible effect of traditional Japanese diet. Cancer Sci, 95, 238-242.

Tyagi B, Manoharm N, Raina V (2010). A case control study on prostate cancer in Delhi. Asian Pac J Cancer Prev, 11, 397-401.

Umesawa M, Iso H, Milami K, et al (2014). Relationship between vegetables and carotene intake and risk of prostate cancer: the JACC study. Br J Cancer, 110, 792-6.

Vlajinac H, Illic M, Marinkovic J, Sipetic S (2010). Nutrition and prostate cancer. $J$ BUON, 15, 698-703.

Zhou XF, Ding ZS, Liu NB (2013). Allium vegetables and risk of prostate cancer: evidence from 132,192 subjects. Asian Pac J Cancer Prev, 14, 4131-4. 